\title{
Building Efficient Multi-level Wireless Sensor Networks with Cluster-based Routing Protocol
}

\author{
Hnin Yu Shwe ${ }^{1}$, ${ }^{1}$ Arun Kumar ${ }^{1}$ and Peter Han Joo Chong ${ }^{2}$ \\ ${ }^{1}$ School of Electrical and Electronic Engineering, Nanyang Technological University, Singapore \\ ${ }^{2}$ Department of Electrical and Electronic Engineering, Auckland University of Technology, New Zealand \\ [email: hninyushwe@ntu.edu.sg, arun0020@ntu.edu.sg and peter.chong@aut.ac.nz] \\ *Corresponding author: Peter Han Joo Chong
}

Received January 20, 2016; revised March 13, 2016; revised April 25, 2016; accepted July 13, 2016; published September 30, 2016

\begin{abstract}
In resource constrained sensor networks, usage of efficient routing protocols can have significant impact on energy dissipation. To save energy, we propose an energy efficient routing protocol. In our approach, which integrates clustering and routing in sensor networks, we perform network coding during data routing in order to achieve additional power savings in the cluster head nodes. Efficacy of the proposed method in terms of the throughput and end-to-end delay is demonstrated through simulation results. Significant network lifetime is also achieved as compared with other techniques.
\end{abstract}

Keywords: clustering, routing, wireless sensor network, energy efficient, building.

A preliminary version of this paper appeared in IEEE VTC 2015, May 11-15, Glasgow, Scotland. 


\section{Introduction}

Wireless sensor networks (WSNs) will play a crucial role in the growth of smart building systems over the next decade. Smart building systems are becoming popular and significant due to the improvement they provide to the quality of life. One of the key components of a smart building system is a sensor network, which provides the necessary information to the smart building system, allowing it to control and monitor the physical environment. Fig. 1 shows a basic architecture of WSN in a smart building and its functioning.

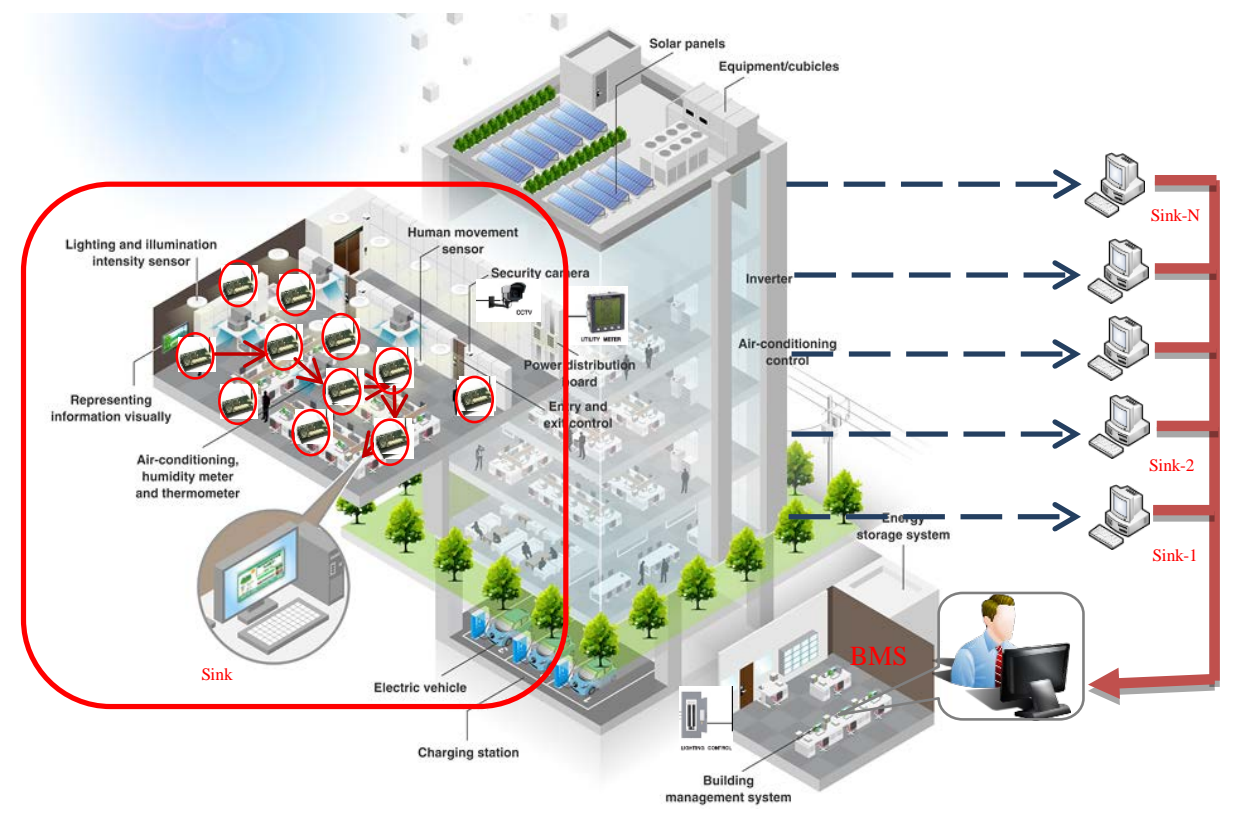

Fig. 1. Wireless sensor network in smart building.

WSNs consist of at least one sink node (or base station) and a (large) number of tiny sensor nodes deployed in the network field. Sensor nodes in the field sense and collect raw data from the environment to do some local processing [1] [2]. If necessary, they also communicate with each other to perform aggregation, and then route the aggregated data to a sink. A sink or base station serves as a destination node for the sensors. Users can access the data from the sink by internet or satellite. The sensor nodes are physically small or tiny devices and are operated by removable battery attached to it. Hence, the sensors have limited capabilities in terms of memory, information sensing, data processing, computation and communication capabilities, and most importantly, they have limited power supply [3].

Due to these limitations of sensor nodes, it is paramount to design energy efficient protocols to prolong the lifetime of the sensor nodes and ultimately the sensor networks. Node clustering and data aggregation are popular techniques to reduce energy consumption in large WSNs [4]. Clustering is the process of dividing the nodes of the sensor network intro groups, where each group has a special node called cluster head $(\mathrm{CH})$. Cluster heads are responsible for gathering the sensory data from all the group members, aggregating it and sending the aggregated data to the base station (BS). Clusters are created using efficient cluster selection algorithms. 
In WSNs, data transmission can either be single hop or multi hop [5]. In either case, data collected by the sensor nodes are sent to the BS to further processing. Thus, routing becomes critical in WSNs. LEACH [6] is one of the most popular routing protocols with cluster-based and hierarchical topology control characters. LEACH can balance the network load, save the energy consumption of communication between nodes and prolong the network life cycle. Several studies have done to enhance LEACH for better network lifetime. S. K. Soni and et.al [24] proposed an enhanced deterministic energy-efficient clustering protocol (EDEC) that determines cluster head election based on the residual energy of each node in WSN and which can significantly prolong the network lifetime. However, these are routing protocols for homogeneous sensor networks and both are not applicable to the real building ambient where heterogeneous sensors are used to obtain building environmental data. According to the characteristics of energy constrained wireless sensor networks, it is more significant to design an energy efficient protocols in practical application. Thus, in this paper, we propose an energy efficient cluster-based routing protocol (EECBRP) to prolong the lifetime of heterogeneous sensor networks in smart building environment.

The rest of the paper is organized as follows. Section 2 introduces the related works. Section 3 briefly presents the proposed energy efficient cluster-based routing protocol. Simulation results and discussions are given in Section 4. Finally, Section 5 concludes the paper.

\section{Related Works}

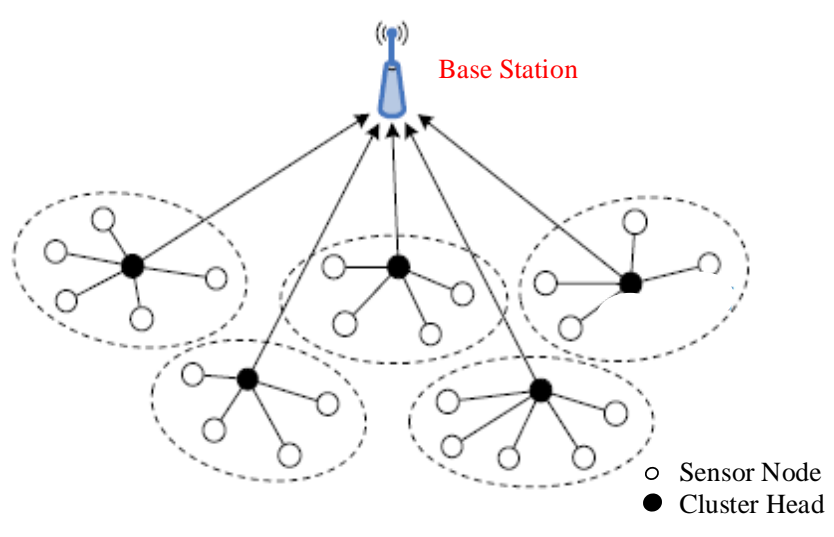

Fig. 2. Architecture of LEACH protocol.

As stated earlier, in WSNs, energy consumption is one of the most important issues and in order to address this issue, a number of routing methods have been proposed in recent years [7-9]. Based on network structure, routing protocols in WSNs can be generally classified into two categories:

1. flat routing topology, and

2. hierarchical routing topology.

In the flat routing topology, all the sensor nodes in the network have the same functionalities and perform the same tasks. Data transmission is mostally performed hop-by-hop in the form of flooding. SPIN [10], TBF [11] and GPSR [12] are some of the well-known flat routing protocols for WSNs. Though, the flat routing protocols are relatively effective in small-scale networks, they are undesirable in large-scale networks. The main 
reason behind this is the large amount of data generated by the sensor nodes which requires large processing power and bandwidth for communication in an already resource-constrained WSNs.

On the other hand, in the hierarchical routing topology, the network is organized into clusters and sensor nodes perform different tasks according to their specific roles. Some best known typical hierarchical clustering routing protocols include LEACH [6], HEED [13] and PEGASIS [14].

Low-Energy Adaptive Clustering Hierarchy (LEACH) is one of the pioneering clustering routing approaches for WSNs. It is a hierarchical protocol in which sensor nodes transmit the sensed data to the $\mathrm{CHs}$, and then the $\mathrm{CHs}$ aggregate and compress the data and directly forward it to the BS as shown in Fig. 2. The main idea of LEACH is to utilize a randomized rotation of local $\mathrm{CH}$ to evenly distribute the energy load among the sensors in the network. And, each node uses a stochastic algorithm at each round to determine whether it can become a $\mathrm{CH}$ in that round. It is assumed that each node has a transmitter radio powerful enough to directly reach the BS. This assumption of direct data transmission to BS requires the full power of the radio and causes energy wastages especially when CHs are far from the BS.

A number of hierarchical protocols based on the principles of LEACH has been proposed in literature such as LEACH-M [15], LEACH-C [16] and so on. The LEACH-M protocol improves the communication mode from single hop to multi-hop between $\mathrm{CH}$ and $\mathrm{BS}$. The LEACH-C uses a centralized algorithm to form clusters. A non-autonomous cluster-head selection is again the main disadvantage of this algorithm.

The proposed routing protocol in this paper falls into the second routing category since hierarchical routing is becoming an active branch of routing technology in WSNs on account of a variety of advantages, such as scalability, data aggregation/fusion, less load, less energy consumption, more robustness, and so on.

\section{Energy Efficient Cluster-based Routing Protocol (EECBRP)}

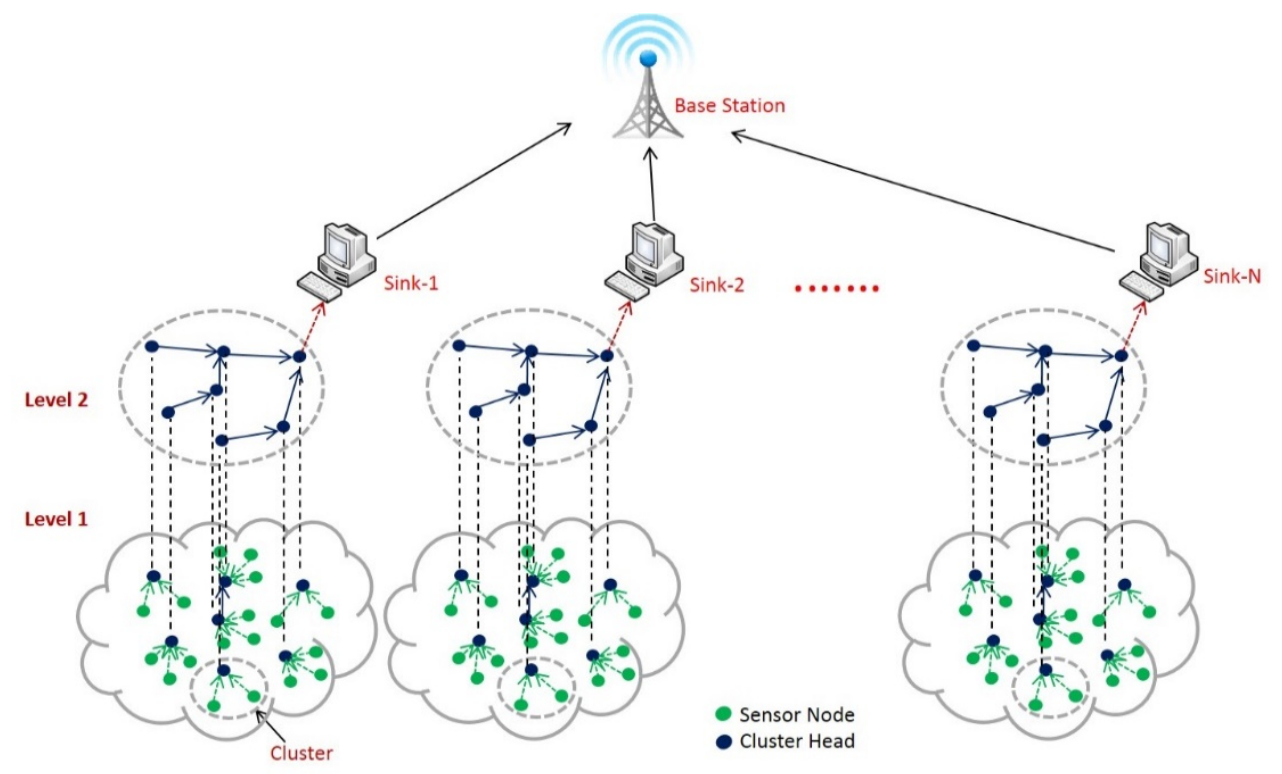




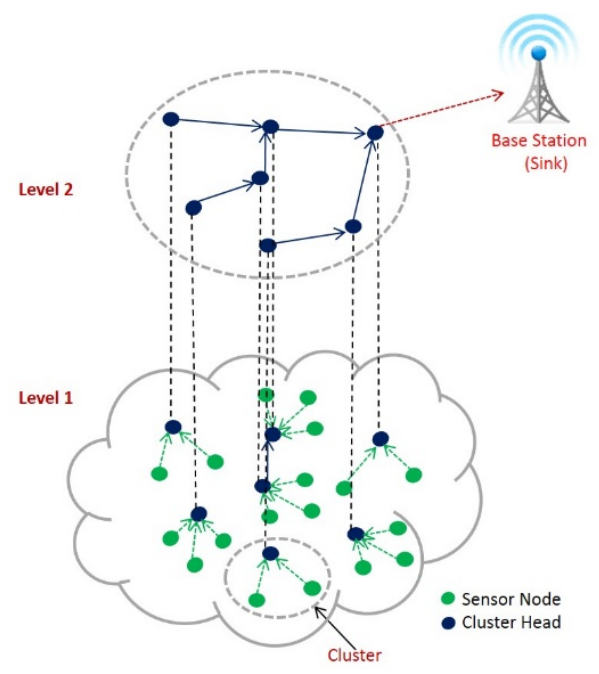

(b)

Fig. 3. Architecture of EECBRP protocol for (a) multi-story (b) single-story building.

In this section, we propose the Energy-Efficient Cluster-Based Routing Protocol (EECBRP) and illustrate the network model of our study. We aim at a smart building automation which not only can help to improve the daily life of the occupants but also can reduce the energy consumption of the building itself. In the building management system as in Fig. 1, a large number of heterogeneous sensor nodes are deployed in various locations to gather the building environmental information and those sensory data are sent to the building automation system to perform the necessary control to the building. The network is divided into a number of clusters and in each cluster a node is selected as a head to perform data collection and aggregation processes inside their respective cluster.

The network architecture of our proposed scheme, EECBRP, for multi-story and single-story buildings are depicted in Fig. 3 (a) and (b) respectively. Fig. 3 (a) depicts multi-story building scenario where each building level is considered to have a separate wireless sensor network and sink node. All the collected sensor readings are first sent to its associated sink via CHs and then the sink will forward the data to the BS. The sink node typically has unlimited power supply and can simply perform the task to forward the data to the BS. Generally, we can consider the data flow in the multi-story building is comparable with the one in single-story building where BS is literally called sink, as in Fig. 3 (b). Here, we logically consider the network as a 2-level network. In level-1, the whole network is broken into set of clusters and member nodes send data to associated $\mathrm{CH}$. In level-2, data communication is carried out only among $\mathrm{CHs}$ in order to forward data to BS (sink).

\subsection{Network Model Assumption}

For the sake of simplicity, we adopt a few reasonable assumptions as follows:

$>$ Sensor nodes are uniformly distributed within a square area.

$>$ Nodes have location information.

$>$ Sensor nodes have same initial energy during their deployment in the network field.

$>$ BS and sensor nodes are static (non-mobile) in nature.

$>$ Sensor nodes are allowed to vary their transmission power. 


\subsection{Proposed Scheme}

Similar with other clustering approaches, the operation of the proposed system is broken up into different rounds. Each round consists of two phases:

1) cluster forming phase, and

2) data routing phase.

In order to rotate the role of cluster head among the network nodes to balance the whole network energy level, the next round to reselect the new CHs will start according to the energy status of the CHs of the current round. The next round will start when the energy of any current $\mathrm{CH}$ becomes less than a certain percentage of its residual energy in the beginning of the round.

1) Cluster Forming Phase

The main task of this cluster forming phase is to choose a $\mathrm{CH}$ and form clusters. The detail algorithm can be seen in Fig. 4.

In the beginning of the cluster forming phase, each node is assigned a unique ID. In literature, there are several mechanisms to assign unique ID to each sensor node in a large-scale sensor networks. The field-wide unique ID schemes, such as GREENWIS and SIDA [17-19], guarantee that there is no ID conflict within the whole application field. Each node is allowed to be uniquely identified and monitored without ambiguity. After assigning node ID, each node broadcasts message which contains its own ID number. In order to decide whether or not to become a $\mathrm{CH}$, each node compares its own ID with the IDs of all receiving messages. If a node finds its ID is greater than the IDs of all receiving messages and its residual energy, $\mathrm{N}_{\mathrm{RE}}$, is higher than the threshold value, $\theta$, it advertises to be a $\mathrm{CH}$ by broadcasting $\mathrm{CH}$-advertise message. Those nodes who received this $\mathrm{CH}$-advertise message will join its cluster by replying $\mathrm{CH}$-associate message. For a node which receives more than one $\mathrm{CH}$-advertise messages, this node will choose the head with the highest received signal strength indicator (RSSI). In cases where the nodes did not receive any $\mathrm{CH}$-advertise messages in a certain period, they declare themselves as $\mathrm{CHs}$ and become isolated clusters. After this phase, the sensor nodes which are having the lowest IDs among the neighbouring nodes are designated as $\mathrm{CHs}$ for the current round and the clusters are organized accordingly.

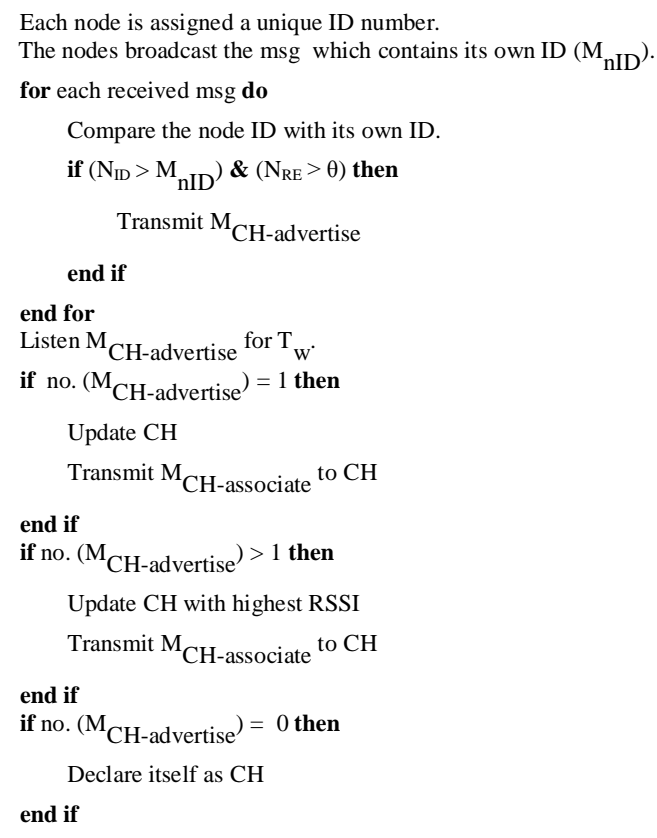

Fig. 4. Clustering algorithm. 
2) Data Routing Phase

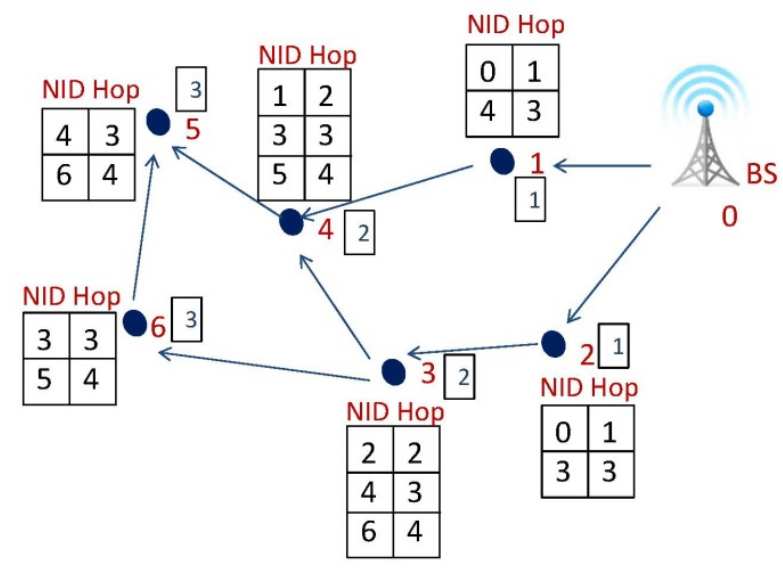

Fig. 5. Neighbour table format.

In our approach, in order to avoid loop-back routing, each $\mathrm{CH}$ maintains a neighbour table which contains information about nodes IDs and hop-to-BS count of neighbouring CHs as shown in Fig. 5. BS initiates the route query message to find out the route in the beginning of the data routing phase. Upon receiving the route query message, each $\mathrm{CH}$ updates the neighbour table with neighbour ID (NID) and hop-to-BS entries. Neighbour tables are constructed in the beginning of the data routing phase and no further update is required in the current round since the network topology remains the same. From this neighbour table, each $\mathrm{CH}$ is able to know its own hop-count information from BS and updates its own hop-to-BS value as well. For example, in Fig. 5, $\mathrm{CH}: 4$ can receive data from its three neighbour CHs; $\mathrm{CH}: 1, \mathrm{CH}: 3$, and $\mathrm{CH}: 5$, where the corresponding hop-to-BS are 2, 3, and 4 respectively. In addition, it can be known that $\mathrm{CH}: 4$ is 2 hop away from BS, which is obtain as the minimum hop-to-BS number in the neighbour table. With the help of this table, each $\mathrm{CH}$ can avoid loop-back data routing by discarding the packets received from lower hop-to-BS nodes. For example, $\mathrm{CH}: 4$ will always discard the packets received from $\mathrm{CH}: 1$ in order to avoid data loop-back.

After constructing neighbour tables, data routing can be carried out. In our proposed approach, we consider heterogeneous radio transmission range for members and CHs. Since member nodes need only inter-cluster communication, they are assigned the transmission range of $15 \mathrm{~m}$ which is also the radius of the cluster. However, CHs need to communicate with neighbouring $\mathrm{CHs}$ for data dissemination; their transmission range is double the transmission range of member nodes. The proposed multi-hop routing works as follow. Each member node sends its sensory data to the associated CH. A simple linear network coding (LNC) [20] is applied in $\mathrm{CH}$ in order to further reduce the energy consumption in data dissemination process. $\mathrm{CH}$ performs LNC upon receiving packets from its member nodes and broadcasts the encoded packets. $\mathrm{CH}$ also performs as a relay node in order to forward the data to the next $\mathrm{CH}$ and the same process is repeated until the data is finally reached to BS. The detail multi-hop routing algorithm can be seen in Fig. 6 . 
1. BS sends the route query msg to the $\mathrm{CH}$ nodes.

2. The nodes updates the neighbor tables with NID \& hop-count upon receiving msg.

3. Each member node sends its data to its associated $\mathrm{CH}$ node.

4. At $\mathrm{CH}$, upon received pkt;

5. if pkt comes from its member then

6. $\quad$ Keep in its buffer, apply LNC \& broadcast encoded pkt.

7. end if

8. if pkt comes from other $\mathrm{CH}$ then

9. Check the NID of incoming pkt.

10. if the pkt is coming from lower hop-count node then

11. Ignore the pkt.

12. else

13. Forward the pkt as it is.

14. end if

15. end if

Fig. 6. Multi-hop routing algorithm.

\subsection{Energy Consumption Model}

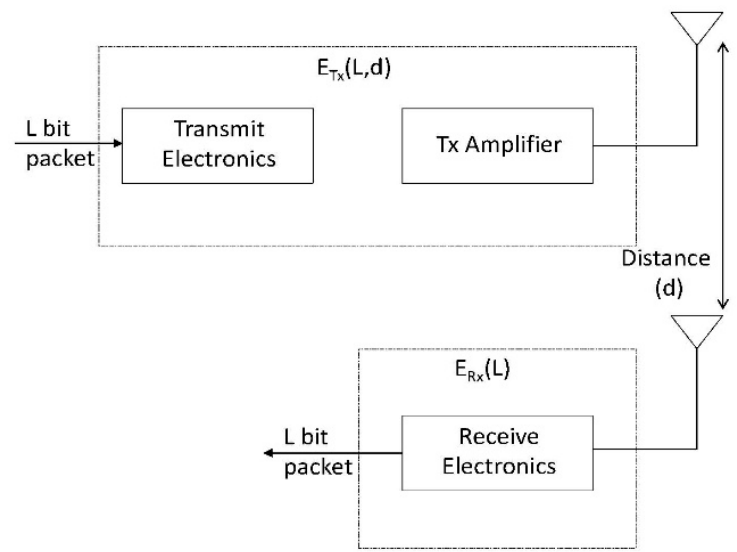

Fig. 7. Radio energy dissipation model.

We use a simplified power model discussed in a number of previous studies [21] [22] [23] [24] for energy dissipation in radio communication. Radio hardware energy dissipation model is shown in Fig. 7. For the sake of simplicity, we first define some parameters used in our model as follows:

$>E_{\text {elec }}$ is the consumed energy per bit.

$>\varepsilon_{f s}$ is the energy consumed by free-space amplifier.

$>\varepsilon_{m p}$ is the energy consumed by multipath amplifier. 
$>d$ is the distance between transmitter and receiver.

$>d_{0}$ is the distance which decide the parameter value of $\varepsilon_{f s}$ and $\varepsilon_{m p}$.

$>E_{N C}$ is the energy required for network coding.

$>L$ is the length of the message in bits

$>$ The round is the time in which all cluster members communicate with CHs and CHs communicate with BS.

Both the free space ( $d^{2}$ power loss) and the multi-path fading ( $d^{4}$ power loss) channel models are used in the model, depending on the distance between the transmitter and receiver. In order to transmit $L$-bit data over distance, $d$, with an acceptable SNR, the amplification energy, $\varepsilon$, is expended to overcome either the free space, $f s$, or multi-path, $m p$, loss, depending on the transmission distance between the two nodes. Therefore, the transmission cost $\left(E_{T X}\right)$ and receiving cost $\left(E_{R X}\right)$ are calculated as follows:

$$
E_{T X}(L, d)=\left\{\begin{array}{l}
L \times E_{\text {elec }}+L \times \varepsilon_{m p} \times d^{2}, \text { if } d<d_{0} \\
L \times E_{\text {elec }}+L \times \varepsilon_{f s} \times d^{4}, \text { if } d \geq d_{0}
\end{array}\right.
$$

where,

$$
E_{R X}(L)=L \times E_{\text {elec }}
$$

$$
d_{0}=\frac{\sqrt{\varepsilon_{f s}}}{\sqrt{\varepsilon_{m p}}}
$$

Therefore, the energy consuming of $\mathrm{CH}_{\mathrm{i}}$ with $n$ members for a round can be calculated as:

$$
\operatorname{Energy}\left(C H_{i}\right)=\sum_{j=1}^{n} E_{R X}\left(L_{j}\right)+E_{N C}(L)+E_{T X}\left(L_{j}, d\right)+E_{S}+E_{I}
$$

In this equation, $n$ is the number of member nodes of $\mathrm{CH}_{\mathrm{i}} ; E_{N C}(L)$ is energy consuming for data encoding with $L$-bits data. $E_{S}$ and $E_{I}$ are the energy consumption in sensing and idle state, respectively.

Hence, the energy consuming of a Non-CH node $m$ in cluster $\mathrm{i}^{\text {th }}$ for a round is:

$$
\operatorname{Energy}\left(\text { Non }-\mathrm{CH}_{m}\right)=E_{T X}\left(L_{m}, d\left(m, C H_{i}\right)\right)+E_{S}+E_{I}
$$

Although actual equipment consumes energy not only when sending and receiving but also while listening, we assume in our model that the listen operation is energy free, since all the evaluated ad-hoc routing protocols will have similar energy consumption due to the node idle time. We further assume a symmetric radio channel; i.e. the same amount of energy is required to transmit a $L$-bit data from node A to B and vice versa.

\section{Performance Evaluation}

In our simulation, we have compared the performance of our proposed algorithm, EECBRP, with the standard hierarchical routing protocol, LEACH, and enhanced deterministic energy-efficient clustering protocol, EDEC. The algorithms are compared with the performance matrices of packet delivery ratio (PDR), end-to-end delay, residual energy, energy dissipation of cluster forming and total number of alive nodes at different round of iterations. 


\subsection{Simulation Setting}

We perform computer simulation using network simulator-2 (NS-2), a standard tool in sensor network simulation. In our simulation, 100 sensor nodes are randomly distributed within a region of $100 \mathrm{~m} \times 100 \mathrm{~m}$ square and BS lies at the corner of this square area. Ignoring the impact of some random factors such as the signal collision and the wireless channel interference, the detail parameter setting for the simulations is shown in Table $\mathbf{1}$.

Table 1. Simulation parameters.

\begin{tabular}{|c|c|}
\hline Parameter Description & Values \\
\hline \hline Simulation Area & $100 \mathrm{~m}^{2}$ \\
\hline Network Size & 100 nodes \\
\hline Placement Model & Random \\
\hline Location of BS & 802.15 .4 \\
\hline MAC Protocol & Constant Bit Rate (CBR) \\
\hline Traffic Type & $15 \mathrm{bytes}$ \\
\hline Data Packet Size & $30 \mathrm{~m}$ \\
\hline Radio Range (non-CH) & Channel/wireless \\
\hline Radio Range (CH) & Antenna/omniantenna \\
\hline Channel Type & $500 \mathrm{~s}$ \\
\hline Antenna Mode & Battery \\
\hline Simulation Time & $2 \mathrm{~J}$ \\
\hline Energy Model & $0.013 \mathrm{pJ} / \mathrm{bit}^{\mathrm{m}} \mathrm{m}^{4}$ \\
\hline Node’s Initial Energy & $100 \mathrm{pJ} / \mathrm{bit} / \mathrm{m}^{2}$ \\
\hline$\varepsilon_{f s}$ & $5 \mathrm{~nJ} / \mathrm{bit}$ \\
\hline$\varepsilon_{m p}$ &
\end{tabular}

\subsection{Simulation Results}

In order to evaluate the performance of our proposed protocol, we computed the residual energy, packet delivery ratio and end-to-end delay under the proposed protocol and compared our proposed scheme, EECBRP, with the standard hierarchical routing protocol, LEACH and energy-efficient clustered protocol, EDEC.

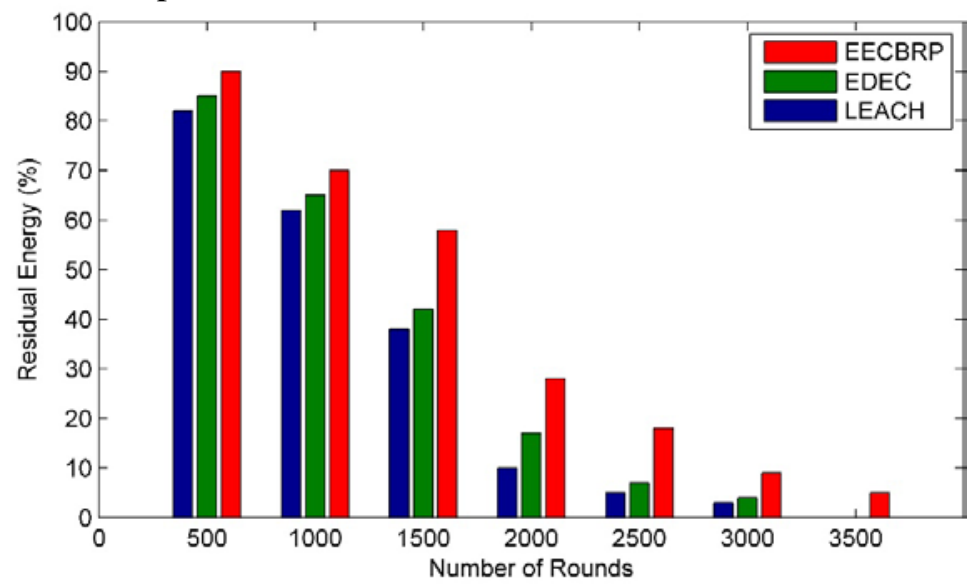

Fig. 8. Residual energy vs. number of rounds. 
Fig. 8 shows the total residual energy within the system against number of rounds, and compares the result of EECBRP with the LEACH and EDEC algorithm. From the figure, it is clear that EECBRP has better energy utilization as compared to LEACH and EDEC algorithms.

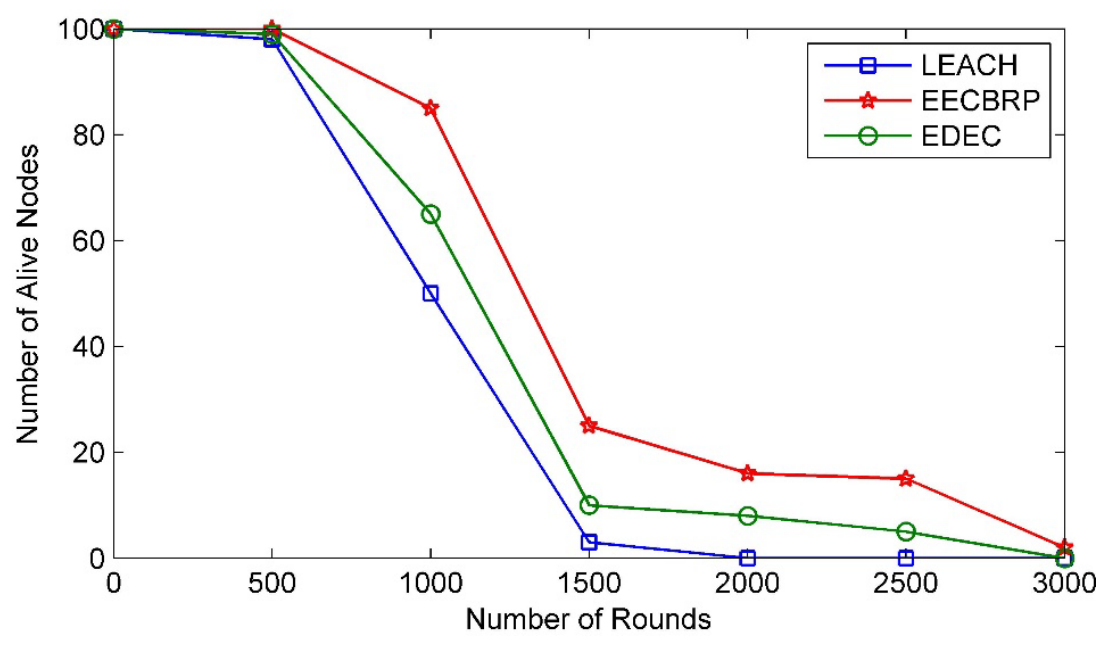

Fig. 9.Number of alive ndoes vs. number of rounds.

Similarly, Fig. 9 shows the number of alive nodes against the number of rounds, and compares the result of EECBRP with the LEACH and EDEC algorithms. It is concluded from the results that EECBRP performs better than both LEACH and EDEC.

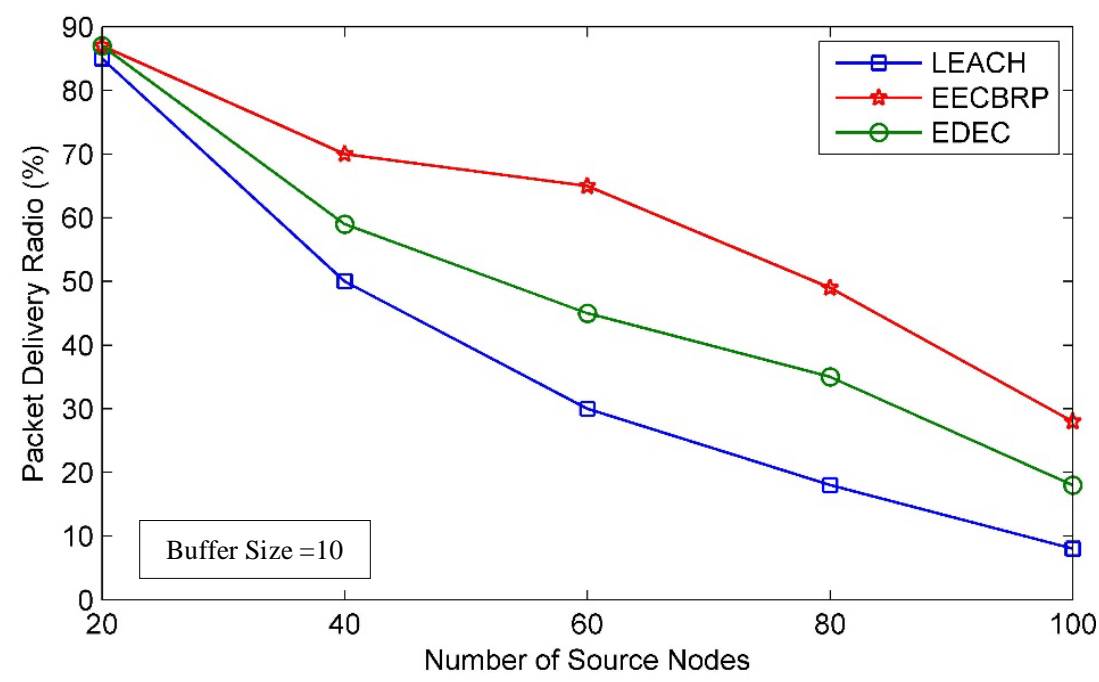

Fig. 10.Packet delivery ratio vs. number of source nodes.

Fig. 10 shows the comparison between the packet delivery ratio and the number of source nodes. From the figure, it is very obvious that the total numbers of packets received by the BS in EECBRP are much greater than the LEACH and EDEC algorithms especially when the number of source nodes are high. 


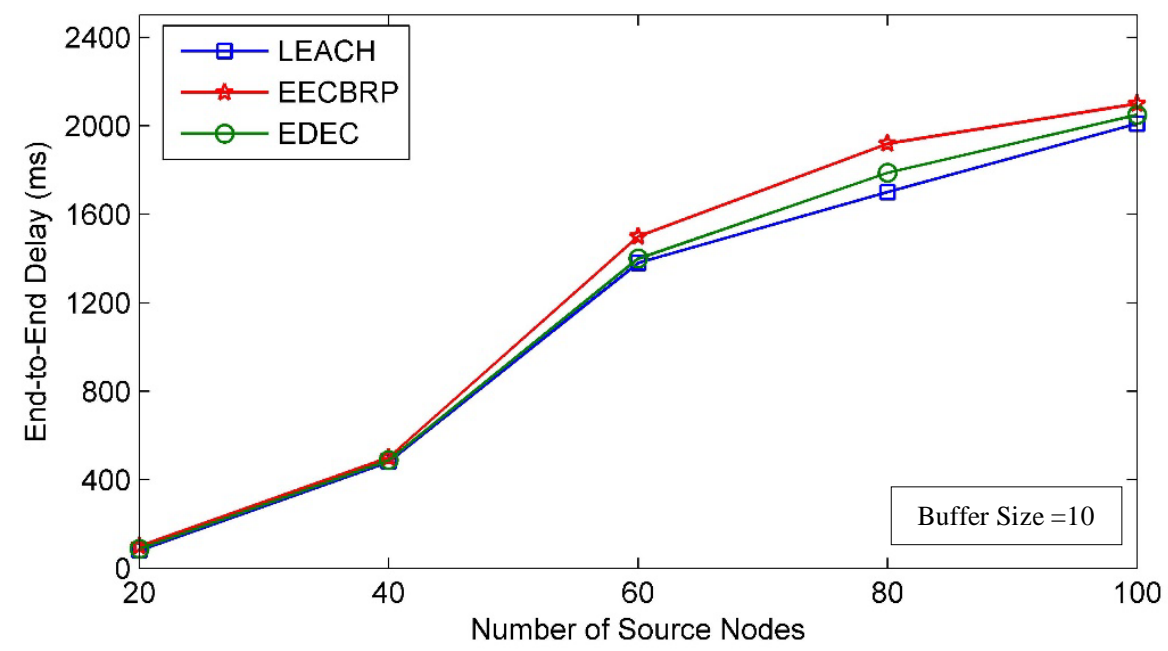

Fig. 11.End-to-end delay vs. number of source nodes.

Fig. 11 shows the end-to-end delay in terms of different number of source nodes. It is clear that the delay of packet delivery from source to sink in case of EECBRP is not much different with the existing LEACH and EDEC algorithms. LEACH slightly outperforms than EECBRP in case of high number of source nodes which is due to the fact that network coding in our algorithm requires some processing time in order to retrieve the original packets. However, this is negligible compare with the energy gain in return.

Table 2. Energy dissipation of cluster forming phase.

\begin{tabular}{|c|c|}
\hline & Energy $(\mathrm{mJ})$ \\
\hline \hline LEACH & 13.5 \\
\hline EDEC & 14.3 \\
\hline EECBRP & 13.8 \\
\hline
\end{tabular}

For the implementation complexity, we finally compare the energy dissipation of cluster forming phase in different approaches. Table. 2 shows the average energy consumptions in cluster forming phase of distributed clustering approaches. Simulation results show our proposed approach, EECBRP, requires less energy than EDEC but slightly more energy than LEACH to setup the clusters. As it can be seen in the table that both EDEC and EECBRP consume slightly more energy than LEACH in setup phase, however, both offer less energy consumption during data routing phase in return.

\section{Conclusion}

In this paper, we have proposed an energy efficient cluster-based routing protocol, EECBRP, for sensor networks. We have performed rigorous tests on the proposed algorithm and experimental results show that the proposed algorithm, EECBRP, is much more efficient than LEACH and EDEC in terms of network lifetime, energy consumption and packet delivery ratio. 


\section{Acknowledgement}

This research is funded by the Republic of Singapore's National Research Foundation through a grant to the Berkeley Education Alliance for Research in Singapore (BEARS) for the Singapore-Berkeley Building Efficiency and Sustainability in the Tropics (SinBerBEST) Program. BEARS has been established by the University of California, Berkeley as a center for intellectual excellence in research and education in Singapore.

\section{References}

[1] C. Ambekar, G. Lakhani, K. Sha and K. Bhanushali, "Energy efficient Data Aggregation in M-SPIN," in Proc. of IEEE 9th International Conference in Intelligent Systems and Control (ISCO), pp.1-5, 9-10 January. 2015. Article(CrossRef Link)

[2] S. Jamil, S. Jamil, S. Ahmed, M. Zubair and F. Sikandar, "COPE: Cooperative Power and Energy-efficient routing protocol for Wireless Sensor Networks," in Proc. of IEEE/ACIS 14th International Conference on Computer and Information Science (ICIS), pp.47-52, June 28-July 1 2015. Article (CrossRef Link)

[3] M. Haenggi, "Opportunities and Challenges in Wireless Sensor Networks," in Proc. of Handbook of Sensor Networks: Compact Wireless and Wired Sensing Systems, CRC Press, Florida, pp. 1.1-1.14, 2004. Article (CrossRef Link)

[4] M. Kuorilehto, M. Hannikainen and T.D. Hamalainen, "A Survey of Application Distribution in Wireless Sensor Networks,” EURASIP Transaction on Wireless Communications Networking, vol. 5, no. 5, pp. 774-788, October 2005. Article (CrossRef Link)

[5] L. S. Shan, Z.P. Dong, L.X. Ke, C.W. Fang and P.S. Liang, "Energy Efficient Multipath Routing Using Network Coding in Wireless Sensor Networks," in Proc. of ADHOC-NOW, LNCS 4104, pp. 114-127, 2006. Article (CrossRef Link)

[6] W. B. Heinzelman, A. P. Chandrakasan, and H. Balakrishnan, "Energy-Efficient Communication Protocol for Wireless Microsensor Networks," in Proc. of the 33rd Hawaii International Conference on System Sciences, January, 2000. Article (CrossRef Link)

[7] P. Maidamwar and N. A. Chvhan, "A Survey on Routing Techniques for Wireless Sensor Networks," in Proc. of National Conference on Innovative Research Trends in Computer Science Engineering Technology, 2012. Article (CrossRef Link)

[8] A. Modirkhazeni, N. Ithnin and M. Abbasi, "Secure Hierarchical Routing Protocols in Wireless Sensor Networks: Security Survey Analysis," in Proc. of IJCCN International Journal of Computer Communications and Networks, vol. 2, Issue 1, February 2012.

[9] K. Guan and L. M. He, "A Novel Energy-Efficient Multipath Routing Protocol for Wireless Sensor Networks," in Proc. of International Conference on Communication and Mobile Computing, pp. 214-218, April 2010. Article (CrossRef Link)

[10] J. Kulik, W. R. Heinzelman and H. Balakrishnan, "Negotiation based protocols for disseminating information in wireless sensor networks,” Wireless Network, vol. 8, pp. 169-185, 2002. Article (CrossRef Link)

[11] D. Niculescu, B. Nath, "Trajectory Based Forwarding and Its Applications," in Proc. of the 9th Annual International Conference on Mobile Computing and Networking (MOBICOM), vol. 8, pp. 169-185, 2002. Article (CrossRef Link)

[12] B. Karp, H. Kung, "GPSR: Greedy Perimeter Stateless Routing for Wireless Networks,” in Proc. of the 6th Annual International Conference on Mobile Computing and Networking (MOBICOM), Boston, MA, USA, vol. 8, pp. 243-254, August, 2000. Article (CrossRef Link)

[13] O. Younis and S. Fahmy, "HEED: A Hybrid, Energy-Efficient, Distributed Clustering Approach for Adhoc Sensor Networks," in Proc. of IEEE Transactions on Mobile Computations, vol. 3, pp. 366-379, 2004. Article (CrossRef Link) 
[14] S. Lindsey, C. Raghavendra and K. M. Sivalingam, "Data Gathering Algorithms in Sensor Networks using Energy Metrics," in Proc. of IEEE Transactions on Parallel Distribution Systems, vol. 13, pp. 924-935, 2002. Article (CrossRef Link)

[15] X. N. Fan and Y. L. Song, "Improvement on LEACH Protocol of Wireless Sensor Networks," in Proc. of the International Conference on Sensor Technologies and Applications, Valencia, pp. 260-264, 2007. Article (CrossRef Link)

[16] W. B. Heinzelman, A. P. Chandrakasan, and H. Balakrishnan, "An Application-specific Protocol Architecture for Wireless Microsensor Networks," in Proc. of IEEE Transactions on Wireless Communications, vol. 1, Issue 4, pp. 660-670, 2002. Article (CrossRef Link)

[17] E. Ould-Ahmed-Vall, D. M. Blough, B. S. Heck, and G. F. Riley, "Distributed Unique Global ID Assignment for Sensor Networks,” Mobile Adhoc and Sensor Systems (MASS), pp. 580-588, 2005. Article (CrossRef Link)

[18] Y. Liu and L. M. Ni, "Location-aware ID Assignment in Wireless Sensor Networks," Mobile Adhoc and Sensor Systems (MASS), pp. 525-529, 2006. Article (CrossRef Link)

[19] J. Lin, Y. Liu and L. M. Ni, "SIDA: Self-organized ID Assignment in Wireless Sensor Networks," Mobile Adhoc and Sensor Systems (MASS), pp. 1-8, 2007. Article (CrossRef Link)

[20] R. Ahlswede, N. Cai, S.Y.R. Li and R.W. Yeung, "Network Information Flow," IEEE Trans. On Info. Theory, vol. 46, pp. 1204-1216, July, 2000. Article (CrossRef Link)

[21] J. G. Jia, Z. W. He, J. M. Kuang and Y. H. Mu, "An Energy Consumption Balanced Clustering Algorithm for Wireless Sensor Network," in Proc. of International Conference on Computational Intelligence and Software Engineering, pp. 1-4, 2010. Article (CrossRef Link)

[22] F. Shang and Y. Lei, "An Energy-Balanced Clustering Routing Algorithm for Wireless Sensor Network,” Wireless Sensor Network, vol. 2, pp. 777-783, October, 2010. Article (CrossRef Link)

[23] F. A. Aderohunmu, J D. Deng and M K. Purvis, "A Deterministic Energy-Efficient Clustering Protocol for Wireless Sensor Networks," in Proc. of Intelligent Sensors, Sensor Networks and Information Processing (ISSNIP), pp. 341-346, 2011. Article (CrossRef Link)

[24] S. K. Soni and G. Kaur, "Performance Analysis of Four Layer Clustering Network Using Enhanced Deterministic Energy-Efficient Clustering Protocol in Wireless Sensor Network," Advanced Computing \& Communication Technologies (ACCT), pp. 679-685, 2015. Article (CrossRef Link) 


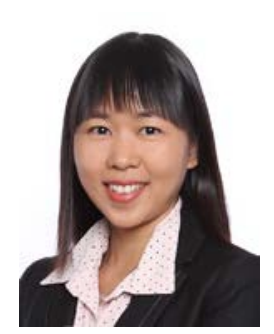

Hnin Yu Shwe received her B.Sc., B.Sc.(Hons), and M.Sc. degrees in 2001, 2002, and 2004 respectively, all from University of Computer Studies, Yangon, Myanmar. She received her Ph.D from the department of Electrical and Communication Engineering, Graduate School of Engineering, TOHOKU University, Japan in 2011. Her research interests include wireless sensor networks, wireless communications, cloud computing, power management, localization, routing and so on. She has published some technical papers in these areas. Currently, she is working as Research Fellow in the department of Electrical and Electronic Engineering, Nanyang Technological University, Singapore.

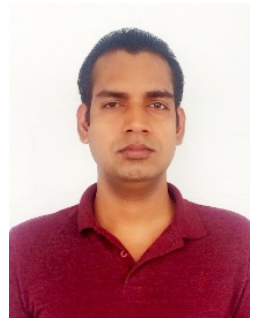

Arun Kumar is a Research Fellow at Electrical Machines and Drives Laboratory in the Department of Electrical and Computer Engineering of National University of Singapore (NUS), Singapore. He has worked as a Post-Doctoral Research Fellow at the Institute of Information Science, Academia Sinica, Taipei, Taiwan from August 2014 to August 2015. He has also worked as a Research Associate at the Infocomm Centre of Excellence (INFINITUS) of the School of Electrical \& Electronics Engineering, Nanyang Technological University (NTU), Singapore. He received his PhD degree from the School of Computer Engineering, Nanyang Technological University (NTU), Singapore in 2014. He received his Master of Technology (M.Tech) Degree in Computer Science \& Engineering from National Institute of Technology, Rourkela in 2008 and the Bachelor of Technology (B.Tech) Degree in Computer Science \& Engineering from Institute of Engineering and Rural Technology (I.E.R.T), Allahabad in 2006. His research interest includes wireless sensor networks, ad-hoc and mobile networks, communication algorithms and computer network analysis.

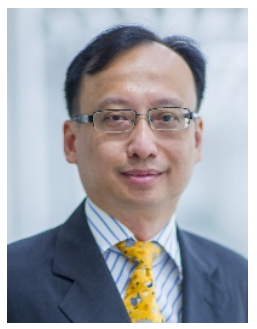

Dr. Peter Han Joo Chong is currently a Professor and Head of Department of Electrical and Electronic Engineering at Auckland University of Technology, Auckland, New Zealand. He received the B.Eng. (with distinction) in Electrical Engineering from the Technical University of Nova Scotia, Halifax, NS, Canada, in 1993, and the M.A.Sc. and Ph.D. degrees in Electrical and Computer Engineering from the University of British Columbia, Vancouver, BC, Canada, in 1996 and 2000, respectively. He has visited Tohoku University, Japan, as a Visiting Scientist in 2010 and Chinese University of Hong Kong (CUHK), Hong Kong, between 2011 and 2012. He is currently an Adjunct Faculty at the Department of Information Engineering, CUHK. He was previously an Associate Professor (tenured) from 2009 to 2016 and Assistant Professor from 2002 to 2009 in the School of Electrical and Electronic Engineering at Nanyang Technological University (NTU), Singapore. Between 2011 and 2013, he was an Assistant Head of Division of Communication Engineering. Between 2013 and 2016, he was a Director of Infinitus, Centre for Infocomm Technology. He was the recipient of 'EEE Teaching Excellence Award' and 'Nanyang Award Excellence in Teaching' in 2010, and 'Nanyang Education Award (College)' in 2015. In 2015, he became a Fellow of the Teaching Excellence Academy in NTU. From February 2001 to May 2002, he was a Research Engineer at Nokia Research Center, Helsinki, Finland. Between July 2000 and January 2001, he worked in the Advanced Networks Division at Agilent Technologies Canada Inc., Vancouver, BC, Canada. He is the Co-Founder of P2 Wireless Technology based in Hong Kong. He is an Editorial Board Member of Security and Communication Networks, Wireless Sensor Network, and an Editor of Far East Journal of Electronics and Communications, and KSII Transactions on Internet and Information Systems. His research interests are in the areas of mobile communications systems including radio resource management, multiple access, MANETs/VANETs, multihop cellular networks and Internet of Things/Vehicles 